ज्ञ FRANÇAISE

$\supset \mathrm{DE}$

띨 PÉDAGGIE

\section{Revue française de pédagogie}

Recherches en éducation

168 | juillet-septembre 2009

Enseignement et apprentissages, entre psychologie et didactiques

\title{
Variations sociales des représentations de l'autonomie dans le travail scolaire chez les collégiens et lycéens
}

Social differences in the representations of autonomy in schoolwork in middle

and high school

Variaciones sociales de las representaciones de la autonomía en las tareas

escolares en los alumnos de colegio y de instituto

Soziale Schwankungen bei der Vorstellung von Selbständigkeit in der

Schularbeit bei 6- bis 12-Klässlern

Rachel Gasparini, Odile Joly-Rissoan et Monique Dalud-Vincent

\section{OpenEdition \\ Journals}

Édition électronique

URL : http://journals.openedition.org/rfp/1791

DOI : 10.4000/rfp. 1791

ISSN : 2105-2913

Éditeur

ENS Éditions

Édition imprimée

Date de publication : 1 juillet 2009

Pagination : 93-109

ISBN : 978-2-7342-1172-3

ISSN : 0556-7807

\section{Référence électronique}

Rachel Gasparini, Odile Joly-Rissoan et Monique Dalud-Vincent, « Variations sociales des

représentations de l'autonomie dans le travail scolaire chez les collégiens et lycéens », Revue française de pédagogie [En ligne], 168 । juillet-septembre 2009, mis en ligne le 01 juillet 2013, consulté le 19 avril 2019. URL : http://journals.openedition.org/rfp/1791; DOI : 10.4000/rfp.1791 


\section{Variations sociales des représentations de l'autonomie dans le travail scolaire chez les collégiens et lycéens \\ Rachel Gasparini, Odile Joly-Rissoan et Monique Dalud-Vincent}

L'autonomie est une compétence socialement et scolairement valorisée. Mais contrairement à ce que laisse penser une conception courante de l'adolescence et de sa "crise », tous les adolescents n'aspirent pas à être autonomes au même âge. L'exploitation secondaire des données de l'enquête "Éducation et famille " (INSEE, 2003) est l'occasion d'observer combien les représentations des collégiens et des lycéens de 10 à 23 ans concernant l'âge auquel on peut faire ou décider seul ne sont pas uniformes : elles sont socialement déterminées (d'après la profession des parents, leur niveau d'études, mais aussi le sexe de l'adolescent) et elles varient également selon les domaines. La question de l'organisation autonome de son travail scolaire paraît particulièrement intéressante à étudier, étant donné l'importance accordée actuellement à l'autonomie de l'élève à l'école.

Descripteurs (TESE) : adolescent, autonomie, devoirs, parents, enseignement secondaire.

'autonomie est associée dans le sens commun à la période de l'adolescence et à l'idée d'une crise potentielle liée à l'évolution psychologique et biologique des individus, révélant le besoin d'indépendance et d'affirmation de soi des enfants, notamment à l'égard de leurs parents. Poussée à l'extrême, cette représentation tend à évacuer les conditions sociales d'expression de l'aspiration à l'autonomie chez les adolescents. Les analyses socio-historiques de N. Elias $(1985,1991,1993)$ permettent d'une part de comprendre pourquoi l'autonomie est devenue progressivement une dimension comportementale valorisée et discriminante dans nos sociétés occi- dentales ; d'autre part de ne pas oublier combien l'autonomie n'existe pas «telle quelle » en s'activant d'elle-même " naturellement » dans le " caractère » des individus : l'intériorisation des normes de comportement doit toujours être contextualisée relativement à des relations intersubjectives et de domination. L'histoire sociale européenne du Moyen Âge au $\mathrm{XIX}^{\mathrm{e}}$ siècle peut s'interpréter dans le sens d'une transformation progressive de la construction de la personnalité, la contrainte extérieure laissant place progressivement à l'auto-contrainte, sous l'effet notamment de la formation étatique et du monopole de la violence légitime. Dans les sociétés occidentales, 
le mode de vie ainsi que les formes d'éducation qui en découlent imposent aux individus de témoigner de leur capacité à se réguler, à contenir leurs pulsions par le contrôle de soi.

L'autonomie est glorifiée au sein de nos sociétés occidentales notamment en ce qui concerne les enfants, alors même que leur indépendance économique ne cesse d'être retardée. Le discours pédagogique contemporain tient l'autonomie pour un bien évident (Hameline, 1999) et l'histoire de la « forme scolaire " (Vincent, 1980) rappelle combien on est passé d'un élève "dressé » à un élève " raisonnable ", qui doit montrer qu'il sait faire seul, qu'il a compris ce qu'il faut faire selon un cadre scolaire déterminé. En France, la loi d'orientation et de programme sur l'avenir de l'école (MEN, 2005) définit les compétences et les connaissances considérées comme indispensables au terme de la scolarité obligatoire pour « accomplir avec succès sa scolarité, poursuivre sa formation, construire son avenir personnel et professionnel et réussir sa vie en société » (article 9). Le socle commun de connaissances et de compétences se déclinait alors en cinq piliers qui ont été complétés suite au décret du 11 juillet 2006 (MEN, 2006) par deux piliers : «compétences sociales et civiques » et « autonomie et initiative » (1).

La notion d'autonomie est devenue incontournable dans le discours des enseignants qui préfèrent ce terme à celui de " discipline » (mot qui leur paraît trop dur et inadéquat aux pratiques actuelles) et qui se plaignent fréquemment du manque de responsabilité, de prise en charge personnelle des élèves (Gasparini, 1998). L'autonomie cristallise un ensemble de caractéristiques valorisées du point de vue scolaire : «Autonomie comme autodiscipline corporelle (savoir contenir ses désirs, se tenir, rester calme, écouter, lever le doigt avant de parler, se mettre au travail sans que l'enseignant ait besoin d'intervenir, s'imposer une régularité dans son travail, son effort, être ordonné...) et comme autodiscipline mentale (savoir faire un exercice tout seul, sans l'aide du maître, sans poser de questions, savoir lire avec les yeux et résoudre par soi-même un problème, savoir se débrouiller dans la réalisation d'un exercice scolaire avec les seules consignes écrites...) 》 (Lahire, 1995, p. 50). Les normes éducatives scolaires actuelles valorisent l'élève " actif ", qui réfléchit et découvre par lui-même, qui sait respecter les règles communes, qui sait s'organiser et faire des choix, voire même s'auto-évaluer (Lahire, 2001). A contrario l'école dévalorise l'élève trop dépendant de la personne de l'enseignant (par exemple pour lui rappeler l'ordre disciplinaire ou apporter une aide cognitive) et trop indépendant à l'égard des règles de vie collective (élève indiscipliné) ou des savoirs enseignés (élève indifférent aux savoirs scolaires).

L'« Enquête permanente sur les conditions de vie des ménages » (EPCV) et sa partie variable "Éducation et famille » (INSEE, 2003, voir la note méthodologique en annexe) (2) livrent des données dont l'exploitation secondaire permet d'analyser les représentations des collégiens et des lycéens de 10 à 23 ans concernant l'âge auquel un " enfant " ou un « jeune » devrait avoir le droit de faire seul ou de décider soi-même dans un certain nombre de domaines : loisirs, sorties, achats, sociabilité, vêtements, heure de coucher, télévision, jeux vidéo, travail scolaire. Partant de l'hypothèse selon laquelle les réponses des adolescents dépendent de la manière dont ils perçoivent leur propre marge de liberté dans le cadre de l'éducation familiale, l'ensemble des réponses et plus particulièrement celles concernant la dimension scolaire (3) ont été analysées en fonction de dimensions sociales inhérentes aux enfants eux-mêmes (âge, sexe) et à leurs parents (catégorie socioprofessionnelle, niveaux d'études).

\section{L'ASPIRATION À L'AUTONOMIE EST PLUS OU MOINS PRÉCOCE SELON LES DOMAINES}

Une première analyse globale des questions F5 à F15 montre que les domaines ne sont pas équivalents dans les représentations des collégiens/lycéens (graphique 1). La distribution des réponses montre une hiérarchisation des domaines où l'aspiration à être autonome est tardive (moyenne d'âge plus élevée) dans certains domaines privés de la vie familiale (" inviter des ami(e)s à la maison sans demander l'avis des parents ", " choisir l'heure de son coucher") alors qu'elle est plus précoce (moyenne d'âge moins élevée) concernant la socialisation extra-familiale (" choisir ses émissions de télé ", "passer la nuit chez des ami(e)s ", " choisir tout seul ses vêtements dans les magasins »). Quant à la question sur l'autonomie dans le travail scolaire (âge auquel on peut avoir le droit de " faire seul ", cf. annexe), elle obtient des réponses significativement faibles relativement aux autres questions (4).

Les représentations de la précocité sont donc hiérarchisées selon les domaines. Les raisons de cette hiérarchisation diffèrent cependant selon les domaines. Les enjeux d'autonomie sont sans doute plus 
Graphique 1. - Caractéristiques de tendance centrale concernant l'âge donné aux questions F5 à F15

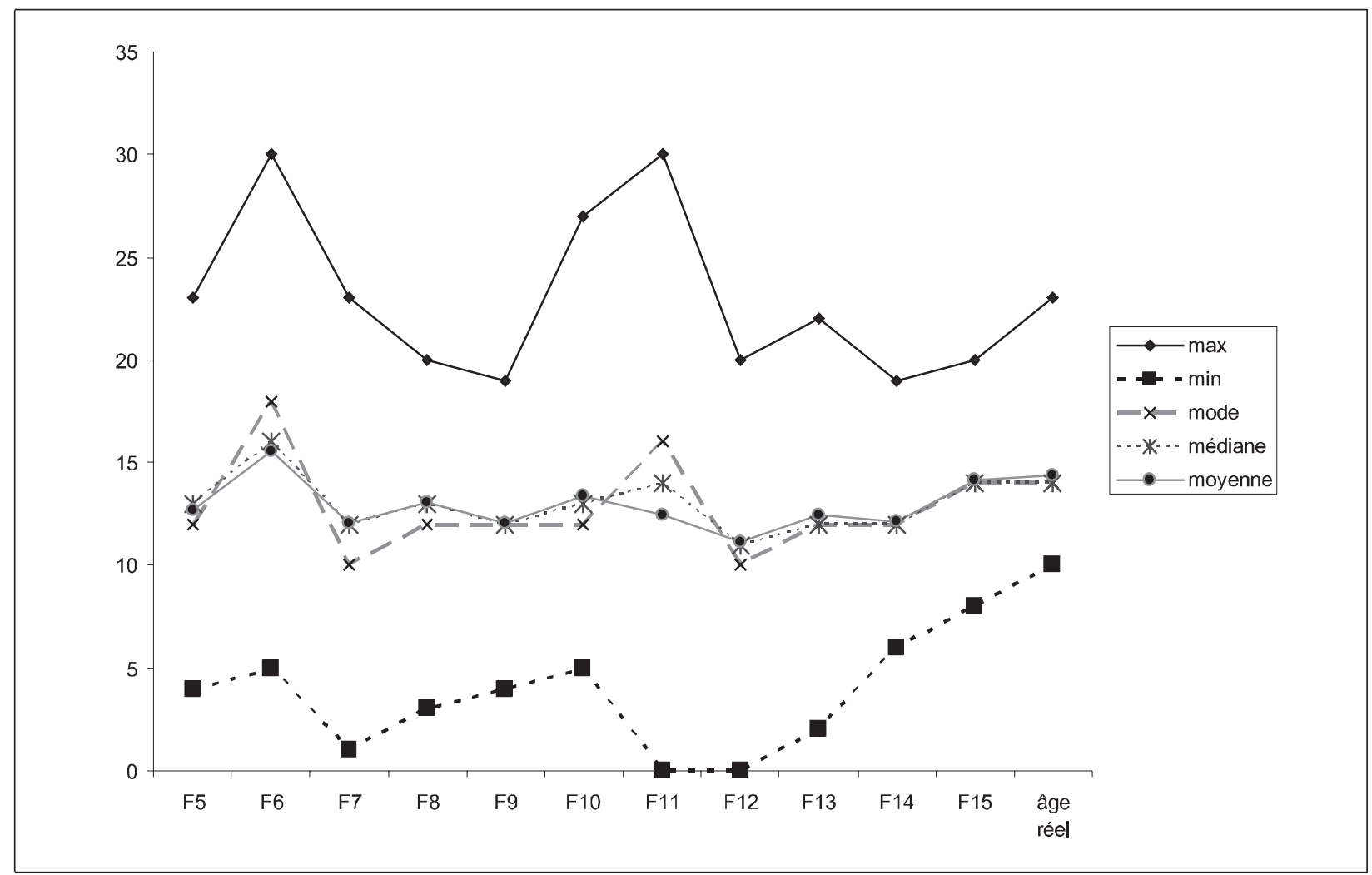

pressés pour ce qui concerne la sociabilité entre pairs que pour ce qui relève de l'organisation privée familiale dans la mesure où l'on peut penser que le groupe de pairs exerce une pression sur la manière de s'habiller (d'où l'importance de choisir seul les vêtements dans les magasins), sur le fait de connaître les émissions de télévision en vogue pour les adolescents (d'où l'importance de pouvoir choisir ces émissions). Par contre, pour le domaine scolaire, il s'agit sans doute d'une injonction institutionnelle correspondant à l'entrée au collège et se poursuivant par l'exigence d'être de plus en plus autonome à mesure qu'on avance dans les classes.

On peut se demander ensuite si les représentations des collégiens/lycéens concernant l'âge auquel on peut faire seul ou décider soi-même ne sont pas liées à leur âge biologique. "Organiser son travail scolaire " est la question qui admet la plus forte corrélation avec l'âge réel (tableau 1), sachant que le lien n'est pas très fort $(0,34)$ mais qu'il indique néanmoins une petite tendance à répondre un âge plus grand quand on est plus vieux, comme si l'élève repoussait «un peu " cette « autonomie » scolaire quand il grandit.

Lorsqu'on regarde la distribution des âges donnés à la question F14: "À ton avis, à quel âge environ un enfant ou un jeune devrait avoir le droit d'organiser son travail scolaire ? » (graphique 2), il s'avère que très peu d'élèves donnent des réponses avant 10 ans et après 16 ans. Les réponses les plus fréquentes se situent entre 10 et 12 ans (l'âge modal se situant à 12 ans), période où les enfants entrent au collège et sont incités encore plus fermement qu'en primaire à développer une attitude autonome à l'égard du travail scolaire, notamment des devoirs à faire le soir à la maison.

D'une manière générale, les élèves ont tendance à donner des âges plus petits que leur âge réel à la question F14 sur l'organisation autonome de son travail scolaire (graphique 3). Ainsi, $25 \%$ des élèves interrogés sont âgés de 12 ans ou moins alors que 
Tableau 1. - Coefficients de corrélation entre les âges donnés aux différentes questions

\begin{tabular}{|l|c|c|c|c|c|c|c|c|c|c|c|c|}
\hline & F5 & F6 & F7 & F8 & F9 & F10 & F11 & F12 & F13 & F14 & F15 & âge \\
\hline F5 & 1 & & & & & & & & & & & \\
\hline F6 & 0,3 & 1 & & & & & & & & & & \\
\hline F7 & $\mathbf{0 , 3 9}$ & 0,33 & 1 & & & & & & & & & \\
\hline F8 & 0,34 & 0,33 & $\mathbf{0 , 4}$ & 1 & & & & & & & & \\
\hline F9 & 0,24 & 0,23 & 0,28 & 0,37 & 1 & & & & & & & \\
\hline F10 & 0,33 & 0,32 & $\mathbf{0 , 3 9}$ & 0,37 & $\mathbf{0 , 4 6}$ & 1 & & & & & & \\
\hline F11 & 0,12 & 0,1 & 0,14 & 0,18 & 0,17 & 0,24 & 1 & & & & & \\
\hline F12 & 0,3 & 0,24 & 0,3 & 0,28 & 0,33 & $\mathbf{0 , 4}$ & 0,24 & 1 & & & & \\
\hline F13 & 0,28 & 0,28 & 0,29 & 0,33 & 0,28 & 0,36 & 0,2 & $\mathbf{0 , 5 9}$ & 1 & & & \\
\hline F14 & 0,34 & 0,2 & 0,32 & 0,28 & 0,27 & 0,34 & 0,23 & $\mathbf{0 , 3 9}$ & $\mathbf{0 , 4 3}$ & 1 & & \\
\hline F15 & 0,29 & 0,35 & 0,23 & 0,27 & 0,26 & 0,27 & 0,12 & 0,3 & 0,33 & 0,38 & 1 & \\
\hline âge réel & 0,16 & $-0,02$ & 0,28 & 0,06 & 0,06 & 0,18 & 0,13 & 0,12 & 0,2 & 0,34 & 0,09 & 1 \\
\hline
\end{tabular}

Remarque : les valeurs en caractères gras indiquent les coefficients les plus forts.

Graphique 2. - Histogramme des fréquences de F14 : "À ton avis, à quel âge environ un enfant ou un jeune devrait avoir le droit d'organiser soi-même son travail scolaire ?"

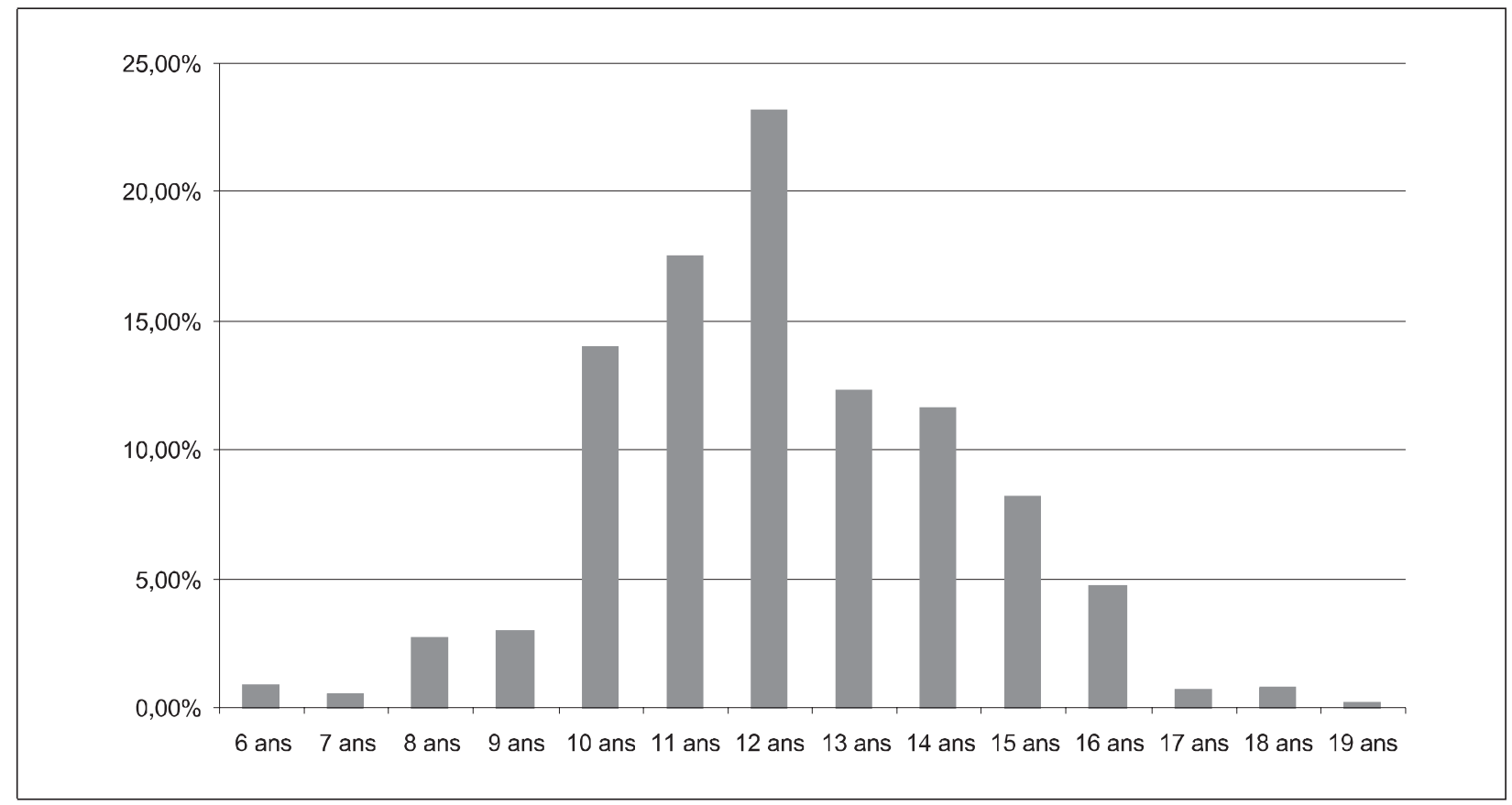


Graphique 3. - Pourcentages cumulés âge réel et F14 : “ À ton avis, à quel âge environ un enfant ou un jeune devrait avoir le droit d'organiser soi-même son travail scolaire ?"

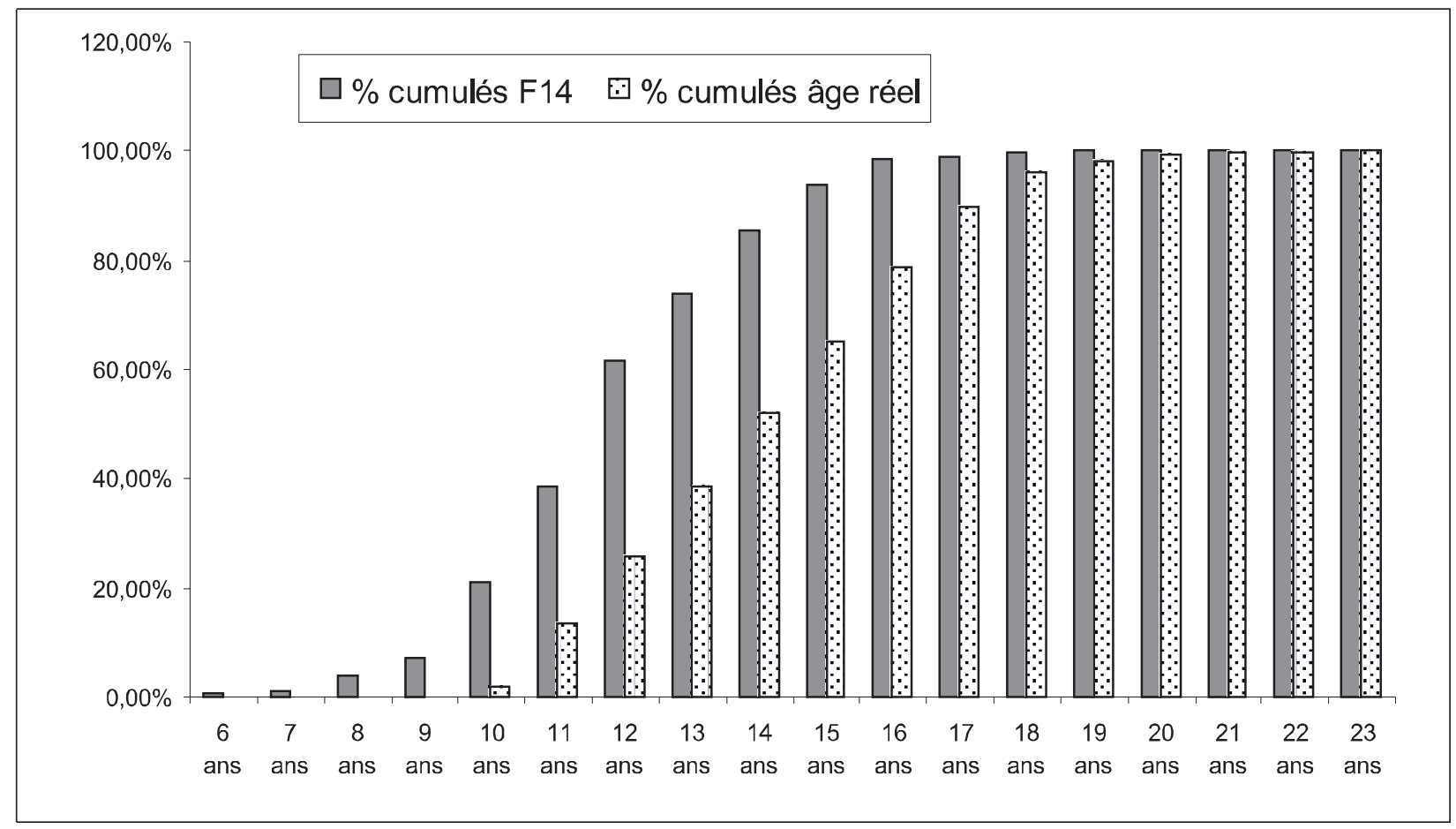

$61 \%$ de cette population ont donné une réponse à F14 valant 12 ans ou moins. II s'agit sans doute d'une anticipation (par les jeunes et par leurs enseignants de cycle 3 en primaire qui préparent les élèves dans ce sens) de la « rupture » dans les modalités de travail que peut représenter l'entrée au collège. Seize ans, période d'entrée au lycée, semble être un âge butoir, puisque c'est pour quasiment tous les jeunes l'âge avant lequel on doit être autonome, même si seulement deux tiers d'entre eux ont 15 ans ou moins. Les années collège semblent donc, aux yeux des adolescents, représenter un enjeu fort d'acquisition de l'autonomie en matière scolaire.

\section{L'AUTONOMIE À L'ÉCOLE}

Avant d'analyser les variations de représentations des collégiens/lycéens en ce qui concerne l'âge auquel on peut organiser soi-même son travail scolaire, il convient de s'interroger sur ce qui fonde la spécificité de l'autonomie scolaire. En effet, l'autono- mie n'est pas une compétence "générale ", transposable quelles que soient les situations et il importe de comprendre le cadre dans lequel elle peut se réaliser avec les normes de comportement qu'il sous-tend. L'analyse socio-historique de la forme scolaire et de ses variantes (Vincent, 1980) souligne les liens entre les pratiques de l'école et le processus de rationalisation dans les sociétés occidentales depuis le $\mathrm{xVI}^{\mathrm{e}}$ siècle, qui aurait entraîné le passage d'un ordre social basé sur la contrainte extérieure à la valorisation de l'intériorisation de la contrainte. Progressivement est apparue dans le courant du XIX $X^{\mathrm{e}}$ siècle l'idée selon laquelle la loi qui était imposée de l'extérieur à l'écolier devait être désormais "intégrée ", "intériorisée » dans la personnalité même de l'écolier. Dire que l'autonomie est valorisée à l'école actuellement ne veut pas dire que les enfants sont libres d'agir et de s'exprimer comme ils le veulent : les règles n'ont pas disparu, c'est le rapport aux règles qui a changé. Réussir scolairement suppose d'avoir compris la forme scolaire d'apprentissage, d'en avoir intériorisé ses règles et non pas de s'en affranchir. La recherche de Nell Keddie (2007) sur la transmission des savoirs 
en histoire, géographie et sciences sociales dans l'enseignement secondaire illustre à quel point les bons élèves sont ceux qui sont prêts à adopter les définitions de l'enseignant et qui prennent de la distance avec les savoirs utilisés dans la vie quotidienne, de manière à pouvoir admettre les sciences sociales comme un nouveau sujet d'études. L'autonomie à l'école est bien spécifique par rapport à d'autres domaines tels que l'habillement, les sorties, la vie familiale : elle est liée au processus d'objectivation des savoirs et à leur organisation scolaire. Les élèves en réussite ne sont pas autonomes uniquement dans leur comportement de "métier d'élève " mais également dans leur travail d'apprenant qui témoigne d'une réelle activité cognitive et d'un travail de décontextualisation et recontextualisation des situations et des activités : les savoirs peuvent être mobilisés dans d'autres contextes, les élèves donnent un sens aux disciplines, sens qui va au-delà des exercices et des activités scolaires, et ils montrent une autonomie relative par rapport au travail et à la personne de l'enseignant (Bautier \& Rochex, 1998). L'autonomie scolaire demandée à l'élève s'exerce donc dans deux pôles : politique (vie collective, règles de vie commune, discipline...) et cognitif (appropriation des savoirs par la lecture silencieuse, la capacité à chercher soi-même, à réaliser seul un exercice à partir des consignes écrites...).

S'il veut organiser seul son travail scolaire efficacement (question F14), l'adolescent doit faire preuve de cette autonomie politique (arriver à se mettre au travail, le faire dans les temps...) et cognitive (en lien avec une culture écrite et des savoirs objectivés scolairement à travers des exercices, des leçons, des activités). II doit parvenir à transposer les obligations de l'école dans un cadre privé, en étant plus ou moins épaulé par son entourage (parents, famille élargie, frères et sœurs...). De ce point de vue, les élèves paraissent inégalement préparés à l'autonomie scolaire. E. Plaisance (1986) a souligné combien la mise en valeur de l'autonomie à l'école maternelle française à partir des années 1950, à côté de l'émergence de valeurs telles que la personnalité de l'enfant et sa coopération, n'est pas neutre socialement : les changements de programme ont caractérisé le passage d'un modèle productif à un modèle expressif qui aurait rendu l'école maternelle moins lisible aux parents de milieux populaires et à leurs enfants. Les enseignants qui exercent en milieux populaires se plaignent souvent de l'absence d'autonomie de leurs élèves : ils doivent veiller à ce que le travail soit bien commencé, à ce que les élèves soient attentifs, à ce qu'ils écoutent, il faut rappeler les consignes, être près d'eux, répondre aux sollicitations, les élèves ne savent pas faire les exercices seuls, se mettre au travail seuls. Les dispositions et compétences prérequises pour les activités scolaires semblent effectivement absentes de certaines configurations familiales (Lahire, 1995) : "Contre un Durkheim par trop rationaliste, on peut dire que l'autonomie n'est pas le fait d'une volonté qui reconnaît la règle comme rationnellement fondée, mais le fait d'un éthos qui reconnaît, immédiatement et tacitement, des principes de socialisation, des règles du jeu pas trop éloignées de celles qui ont présidé à sa propre production » (p. 56). L'autonomie est mise en question par des élèves qui n'ont pas fait " leurs » (auto) "les lois" (nomos) scolaires. Or pour effectuer seul certaines activités, il faut au préalable avoir intériorisé les modalités de certains comportements avec l'aide de l'adulte et les enfants sont inégalement étayés de ce point de vue. Ainsi, dans les familles populaires dont l'enfant est en " rupture scolaire ", il n'est pas rare que les horaires de travail des parents (par exemple les trois-huit ou la flexibilité des heures...) ne permettent pas de réguler celles des adolescents qui doivent intérioriser un rapport au temps propice à la ponctualité, comme se coucher à des heures « raisonnables " ou se lever à temps pour aller en cours (Millet \& Thin, 2005). L'autonomie ne se pose pas pour ces adolescents en termes de " choix " à être autonome mais plutôt d'« obligation " à se débrouiller seul pour anticiper, adopter un rythme en adéquation avec les exigences scolaires.

Au total, les aspirations à l'autonomie scolaire telles que nous pouvons les appréhender à travers les indications des âges à partir desquels on peut organiser seul son travail scolaire doivent être resituées socialement (en appréhendant la plus ou moins grande proximité des parents avec l'univers scolaire, selon leur niveau d'études et leur appartenance socioprofessionnelle) et relativement aux représentations dominantes dans la société (notamment celles liées au genre (5)).

\section{DES VARIATIONS SELON LES FILLES ET LES GARÇONS}

D'une manière générale, les corrélations entre les âges donnés par les garçons aux questions F5 à F15 diffèrent peu de celles obtenues pour les filles (d'après les coefficients de corrélation entre les variables concernées). Sur les onze domaines concernés, 
Graphique 4. - Histogramme des fréquences de réponse pour les filles

et les garçons à la question F14 : "À ton avis, à quel âge environ un enfant

ou un jeune devrait avoir le droit d'organiser soi-même son travail scolaire ?"

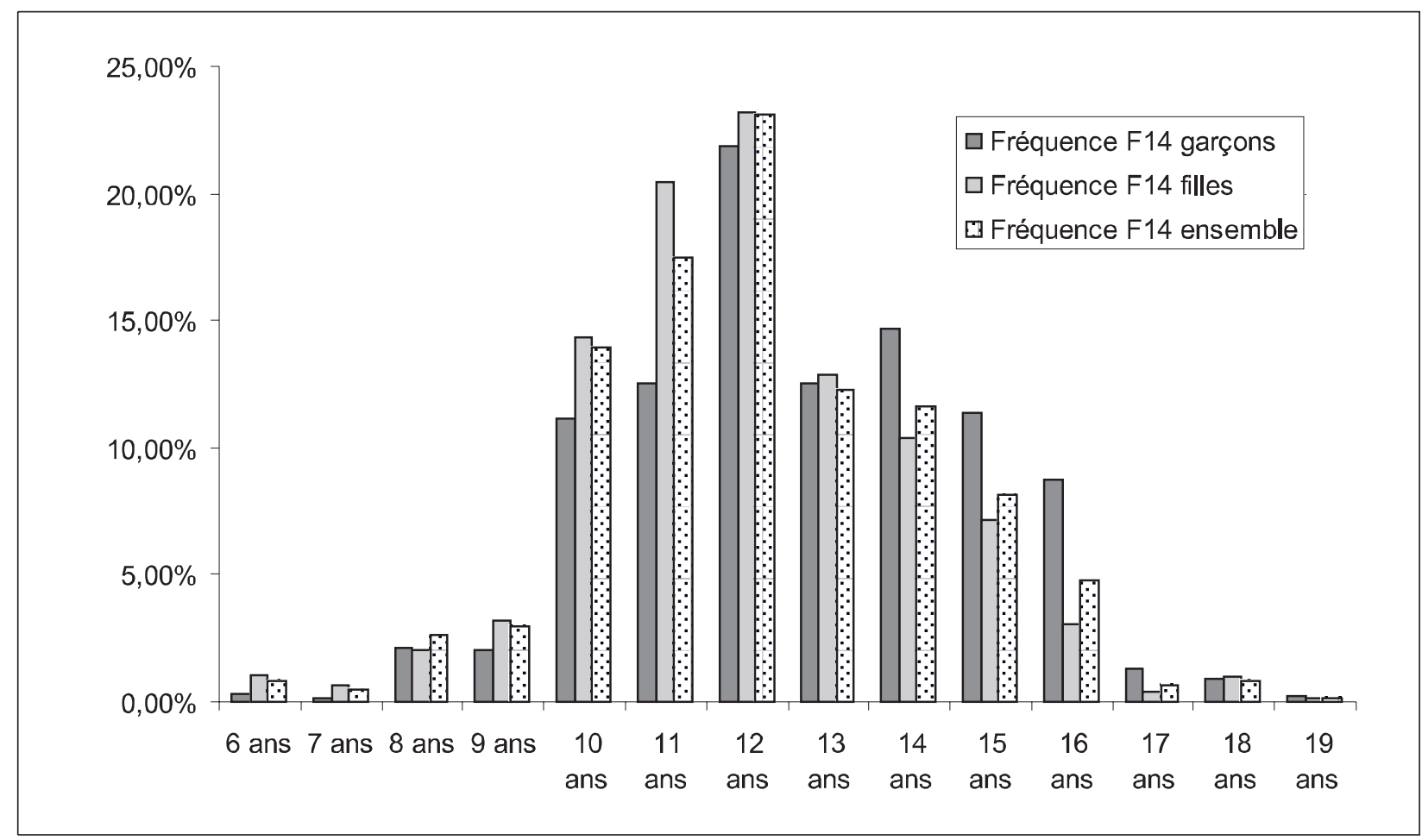

d'après le test approprié de Mann-Withney, les garçons ont des aspirations à l'autonomie plus tardives que les filles dans quatre situations seulement : « organiser soi-même son travail scolaire » (F14), " décider de l'heure de son coucher " (F15), " passer la nuit chez des ami(e)s" (F7) et " choisir seul ses vêtements dans les magasins »(F9). Les variables F14 et F15 sont relativement corrélées entre elles, par rapport aux autres variables, et on peut avancer l'hypothèse que les garçons se sentent capables d'être « responsables » dans ces deux domaines (scolaire, heure de coucher) moins précocement que les filles, sans oublier que ces représentations adolescentes reflètent aussi en partie les convictions sociales concernant la « maturité » des enfants selon leur sexe.

La comparaison de la distribution des âges donnés à la question F14 sur l'organisation autonome de son travail scolaire selon les filles et les garçons (graphique 4) souligne bien que si, pour tous, 10 ans semble comme un âge charnière où l'organisation de son tra- vail scolaire apparaît progressivement comme devant être autonome (12 ans étant un pic), par contre les garçons indiquent plus fréquemment 14,15 et même 16 ans, comme s'ils étaient plus nombreux que les filles à ne pas intégrer cette injonction scolaire à être autonome "dans les temps requis ". Les filles, de leur côté, sont plus nombreuses à répondre 10,11 , 12 ans. Les conditions de scolarisation au collège (multiplication des enseignants, planification du travail par l'intermédiaire d'un cahier de texte, distanciation par rapport aux enseignants et au rôle de l'élève (6)) sont sans doute davantage propices à l'expression, de la part des filles, de leur autonomie dans ce domaine.

Des travaux antérieurs ont montré combien la scolarité des garçons est plus contrôlée par les parents alors que le suivi de la scolarité des filles serait plus diffus, reposant davantage sur la confiance (DuruBellat, 1994, 1995). La prime éducation des filles les prépare à une forme de «docilité » scolairement requise, qui se traduit chez les parents par une 
Tableau 2. - Réponse, selon le sexe de l'enfant, à la question : "Vous arrive-t-il d'être en désaccord avec votre enfant à propos de l'école, de ses études ?"

\begin{tabular}{|l|c|c|c|}
\hline & garçons & filles & Ensemble \\
\hline oui & $57,1 \%$ & $42,9 \%$ & $100 \%$ \\
\hline non & $44,1 \%$ & $55,9 \%$ & $100 \%$ \\
\hline sans objet & $43,7 \%$ & $56,3 \%$ & $100 \%$ \\
\hline Ensemble & $46,8 \%$ & $53,2 \%$ & $100 \%$ \\
\hline
\end{tabular}

Légende : khi $2=19,02 ; \mathrm{ddl}=2$ (significatif à $1 \%)$. Remarque : la modalité "sans objet " signifie que le parent répondant estime que « l'enfant est trop jeune pour aborder un tel sujet ».

attitude plus confiante envers les filles en matière de travail scolaire (concernant les devoirs (7), le contrôle du temps passé devant la télévision) et qui s'exprime par davantage de respect du métier d'élève chez les filles (faire preuve d'assiduité, apporter le matériel requis, respecter le temps scolaire : arriver à l'heure, rendre son travail dans les temps, etc., voir Baudelot \& Establet, 2007).

$\mathrm{Si}$, quand on regarde les données de l'enquête INSEE (2003), le domaine scolaire est selon les parents un objet de conflits plus fréquents chez les garçons que chez les filles (tableau 2), cette distinction ne se traduit pour autant pas chez les adolescents par une perception différenciée de la manière dont ils sont aidés par leurs parents. Les écarts ne sont pas statistiquement significatifs selon le sexe de l'enfant pour les questions portant sur l'aide aux devoirs et sur le suivi des résultats scolaires par les parents, mais on observe cependant une tendance : les filles seraient un peu plus satisfaites que les garçons de l'intervention parentale, notamment paternelle.

\section{DES VARIATIONS SELON LE NIVEAU D'ÉTUDES DES PARENTS}

D'une manière générale, dans l'enquête INSEE, le niveau d'études des parents joue un rôle dans les représentations adolescentes : les enfants des parents les moins diplômés (8) ont une tendance générale à donner un âge plus élevé à la question " À quel âge un enfant ou un jeune devrait avoir le droit de faire les choses suivantes... » (test de Mann-
Withney sur données non appariées). II est possible que ce résultat soit l'effet d'une éducation basée sur un contrôle plus direct dans les milieux populaires que chez les cadres supérieurs, si l'on en croit les analyses de Kellerhals et Montandon (1991) sur les pratiques éducatives parentales. Si cette hypothèse ne doit pas être écartée de nos interprétations, elle ne peut cependant pas être vérifiée avec les données de la présente enquête INSEE, puisque la seule question renseignant sur le style d'éducation comporte des ambiguïtés dans la formulation : « D'une manière générale, penses-tu que tes parents sont : très stricts, plutôt stricts, plutôt souples, très souples ? " Les enquêtés peuvent avoir interprété différemment les notions de «strict " et « souple » qui peuvent concerner les modalités du contrôle (plus ou moins physique, direct) mais aussi l'étendue de ce contrôle (surveiller tous les domaines de la vie de l'adolescent ou certains seulement).

En ce qui concerne les niveaux d'études des parents, le diplôme du père est moins discriminant que celui de la mère quant aux aspirations à l'autonomie des jeunes (respectivement $30 \%$ et $51 \%$ des dominations observées quand on compare groupe par groupe selon le niveau d'études des parents, pour voir quel groupe a tendance à donner des âges plus élevés que l'autre) et on sait par ailleurs que le niveau d'études de la mère agit comme une variable plus explicative de la réussite scolaire des collégiens que le niveau d'études du père (Henri-Panabière, 2007). Les mères dans l'ensemble s'occupent davantage de l'éducation des enfants, notamment de leur scolarité, comme le rappelle l'exploitation des données de la même enquête par Gouyon (2004) : en 2003, 90 \% d'entre elles disent suivre leur enfant en $4^{\mathrm{e}}$. Les parents se sentent inégalement compétents, cette impression étant liée à leur niveau scolaire : $81 \%$ des mères n'ayant aucun diplôme déclarent se sentir " assez souvent dépassées " ou « très souvent dépassées " pour aider leur enfant collégien et, au lycée, ce sentiment est partagé par plus de $90 \%$ des mères ayant au plus le certificat d'études primaires. A contrario $70 \%$ des bacheliers et $90 \%$ des bachelières s'occupent du travail scolaire de l'enfant contre $50 \%$ des hommes et $75 \%$ des femmes ayant au plus un certificat d'études primaires.

Les réponses des collégiens et lycéens sont globalement en phase avec les déclarations de leurs parents. D'une part, plus le diplôme est élevé, plus les parents aident leurs enfants pour les devoirs (graphiques $5 a, 5 b, 6 a$ et $6 b$ ). D'autre part, les mères s'occupent davantage du travail scolaire en général 
Graphique 5a. - Estimation des adolescents concernant le suivi scolaire du père, selon son niveau d'études : "Penses-tu que ton père s'occupe suffisamment de ton travail scolaire ?"

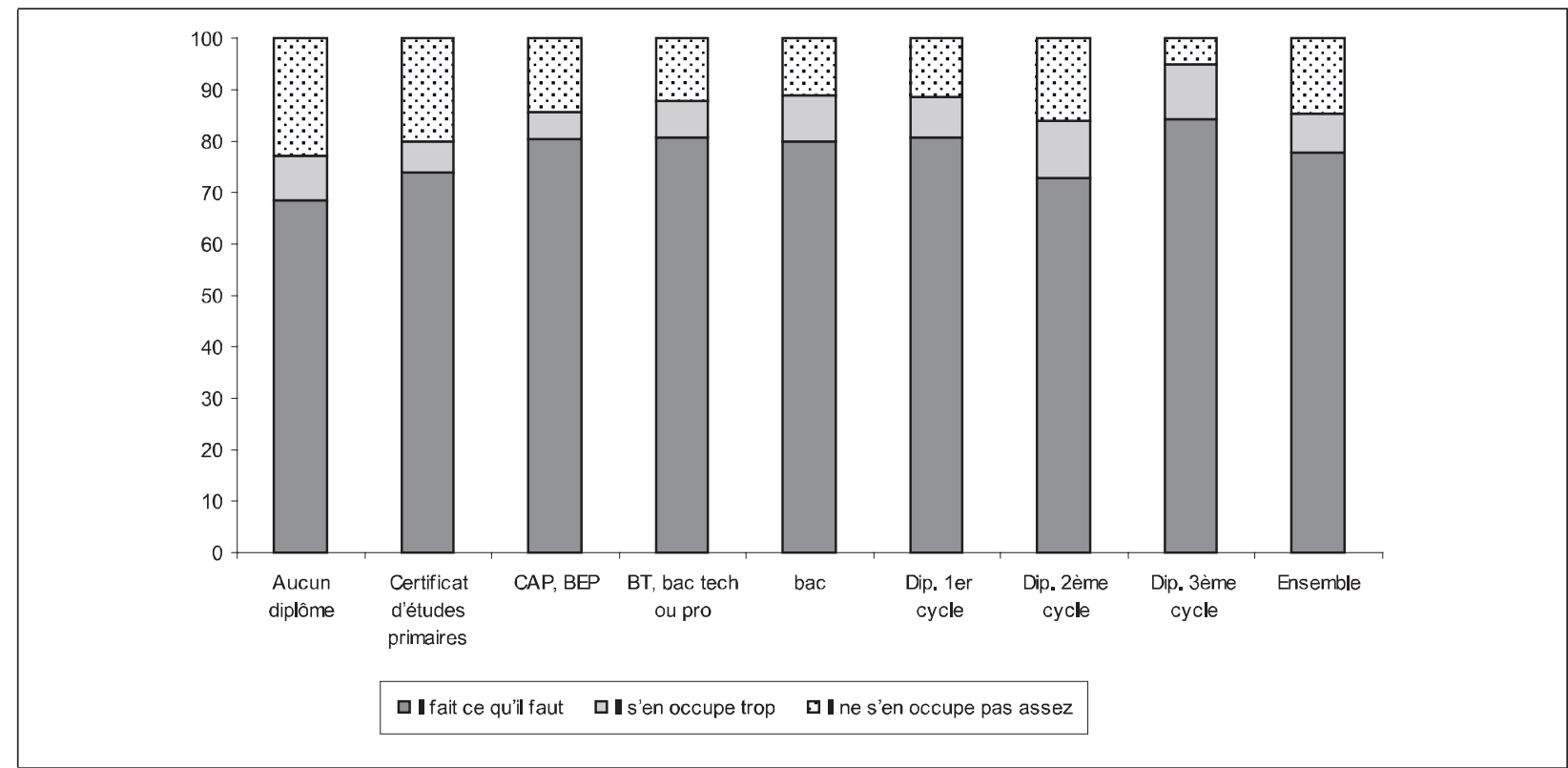

Note : a été enlevée la modalité "sans objet" (père absent du foyer) dont les effectifs sont trop faibles. Champ : collégiens et lycéens de 10 à 23 ans. Source : enquête PCV, partie « Éducation et famille » (INSEE, 2003).

Graphique 5b. - Estimation des adolescents concernant le suivi scolaire de la mère, selon son niveau d'études : «Penses-tu que ta mère s'occupe suffisamment de ton travail scolaire ?"

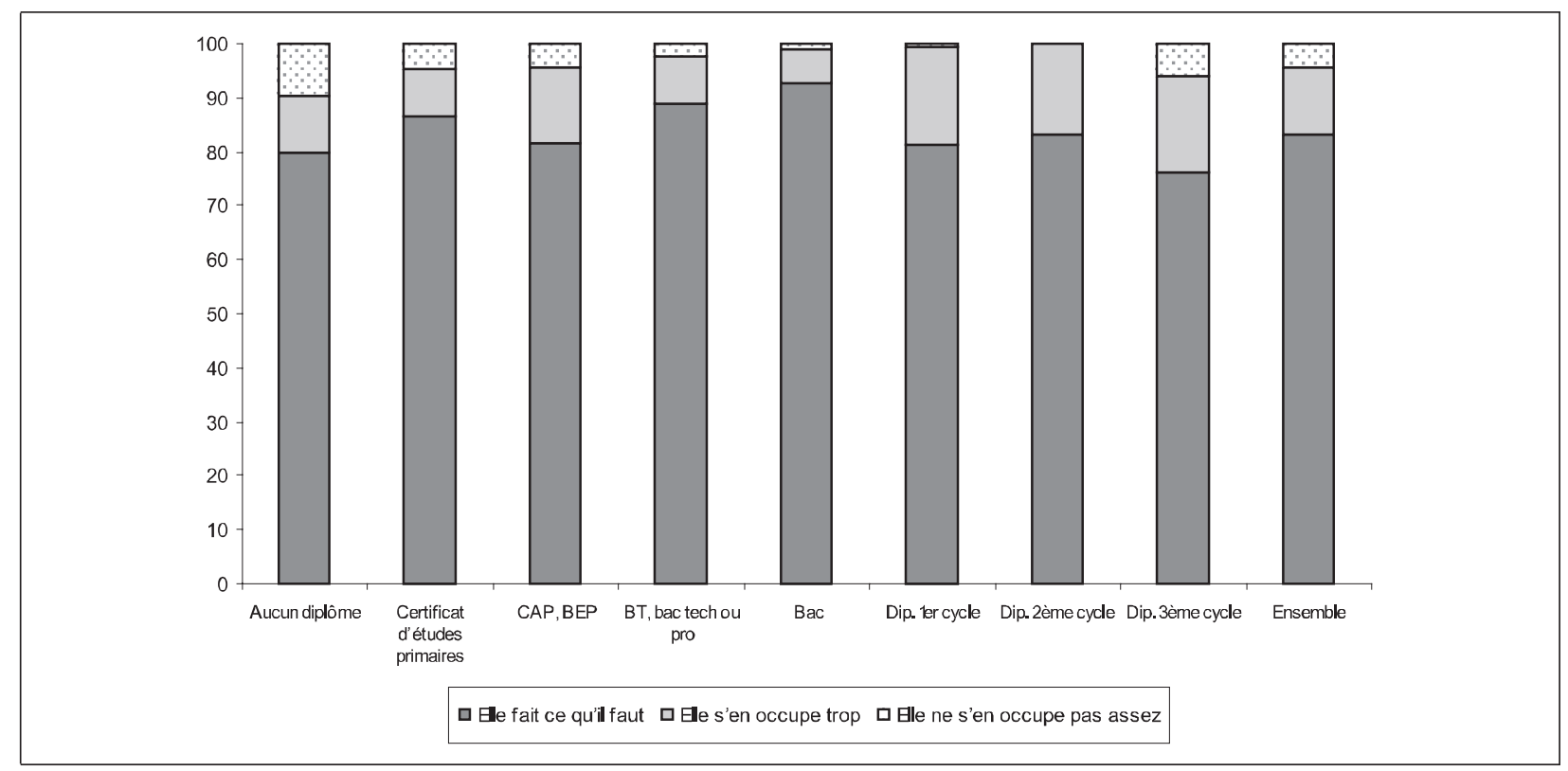

Note : a été enlevée la modalité "sans objet " (mère absente du foyer) dont les effectifs sont trop faibles. Champ : collégiens et lycéens de 10 à 23 ans. Source : enquête PCV, partie «Éducation et famille » (INSEE, 2003). 
Graphique 6a. - Estimation des adolescents concernant l'aide aux devoirs du père selon son niveau d'études : "Si tu as du travail scolaire à faire à la maison, es-tu aidé par ton père ?"

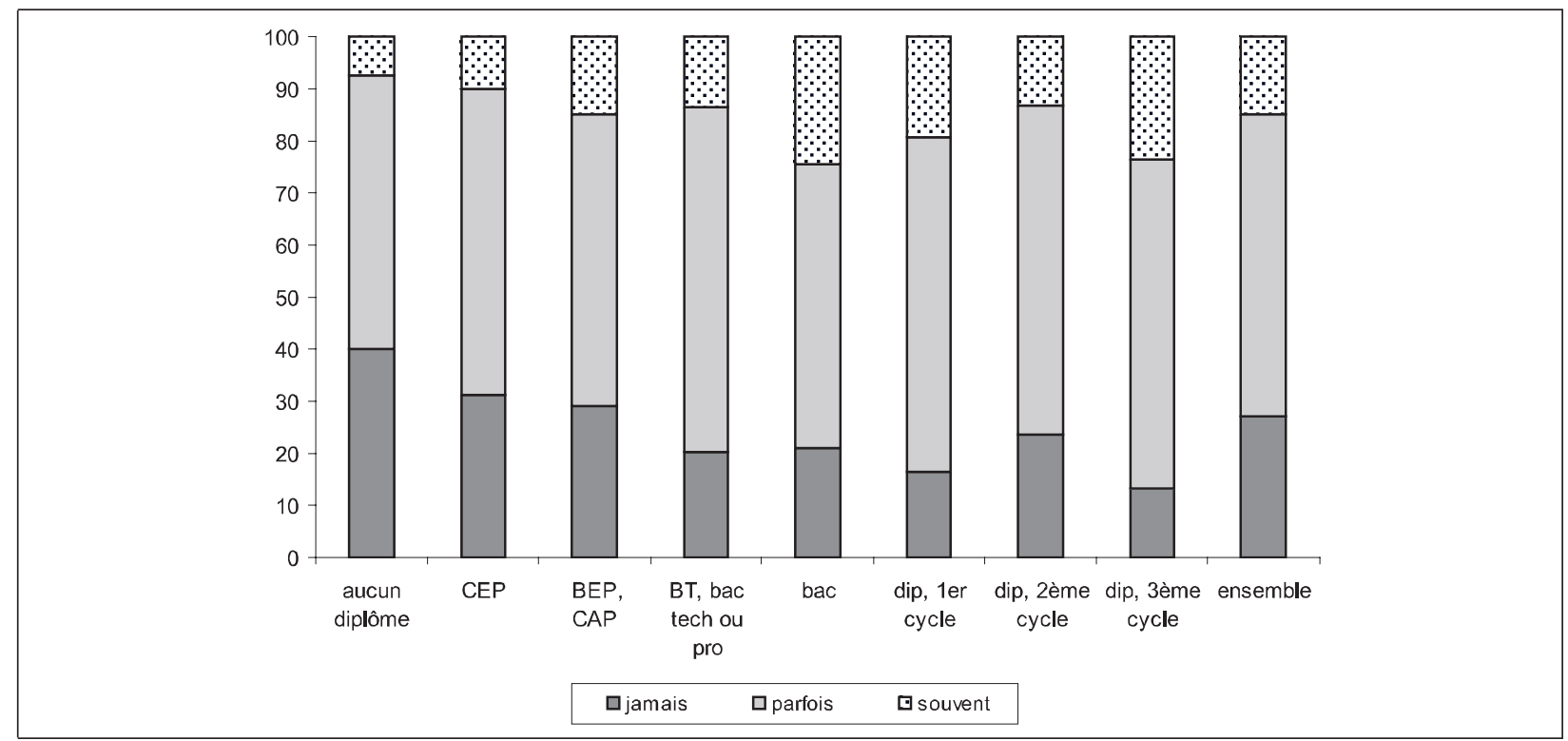

Légende : khi $2=48,4$; ddl = 14 (significatif à $1 \%$ ). Champ : collégiens et lycéens de 10 à 23 ans. Source : enquête PCV, partie " Éducation et famille » (INSEE, 2003).

Graphique 6b. - Estimation des adolescents concernant l'aide aux devoirs de la mère selon son niveau d'études : "Si tu as du travail scolaire à faire à la maison, es-tu aidé par ta mère ? "

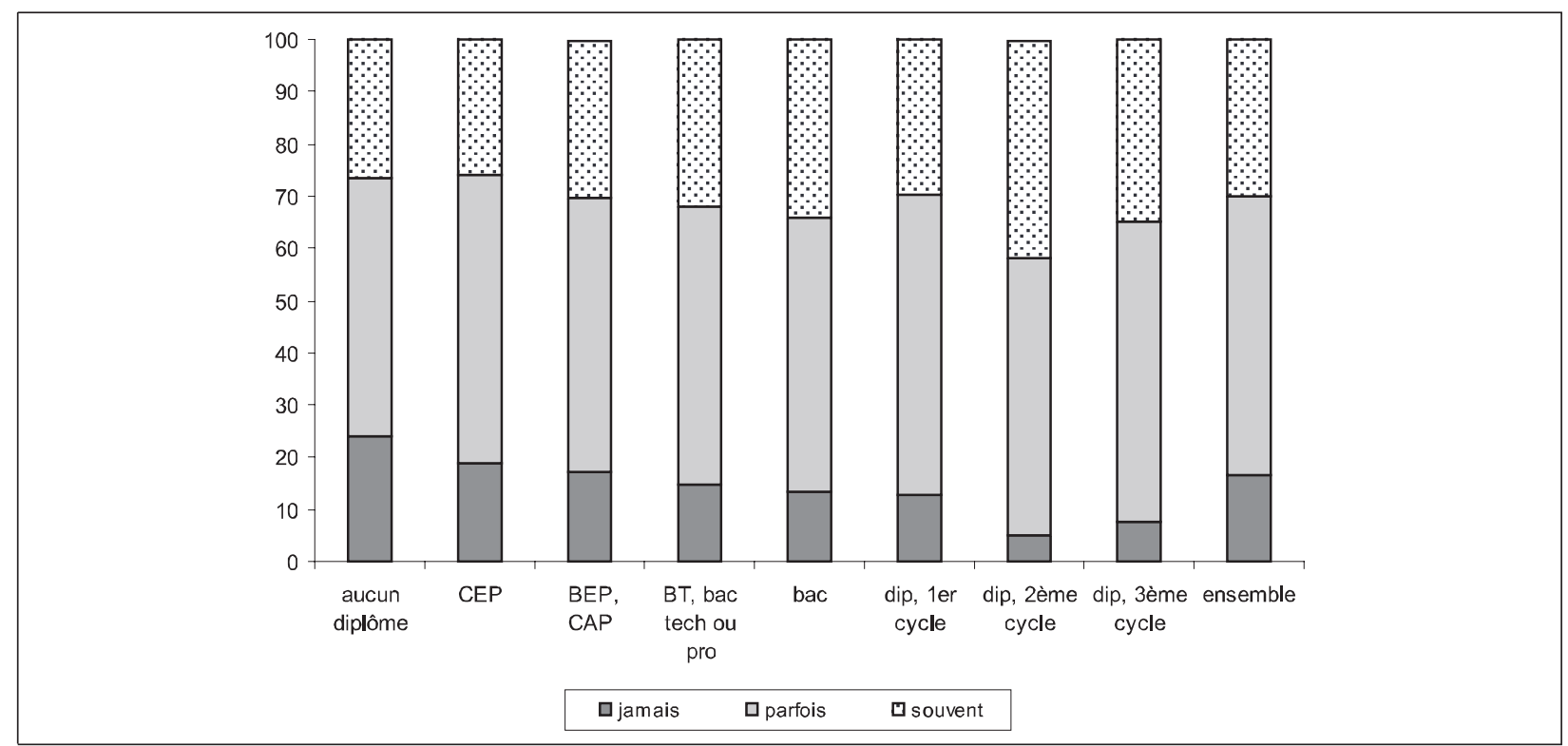

Légende : khi 2 = 25,88; ddl = 14 (significatif à $5 \%$ ). Champ : collégiens et lycéens de 10 à 23 ans. Source : enquête PCV, partie « Éducation et famille » (INSEE, 2003). 
ainsi que des résultats (voir les questions en annexe) : $10 \%$ des adolescents estiment que leurs pères ne surveillent pas les résultats scolaires (contre plus de $2 \%$ pour les mères) et près de $60 \%$ estiment qu'ils s'en occupent « de toute façon » (contre $80 \%$ pour les mères). Enfin, à la question F14 concernant le fait d' " organiser soi-même son travail scolaire ", les enfants dont le père n'a pas de diplôme ont tendance à donner des âges plus élevés que ceux dont le père est doté au moins du CAP/BEP, mais les enfants dont le père a ce niveau n'ont pas tendance à donner des âges plus élevés que ceux dont le père a un niveau d'études encore supérieur. À la même question, les enfants ont des représentations moins souvent précoces si leurs mères sont moins diplômées (et pas seulement si elles n'ont pas de diplôme, comme pour les pères), surtout par rapport aux diplômes universitaires.

Dans le détail, des nuances sont à apporter. Les adolescents dont les pères sont les plus faiblement dotés en capital scolaire (au plus le certificat d'études primaires) se montrent les plus insatisfaits de leur suivi (modalité "il ne s'en occupe pas assez ") alors que la proportion de réponses à la modalité « il fait ce qu'il faut » augmente quasi proportionnellement au niveau d'études paternel (9) (graphique 5a). Par contre concernant les mères, l'estimation des adolescents qu'elles font « ce qu'il faut " par rapport à leur travail scolaire ne varie pas proportionnellement au niveau d'études maternel : les plus satisfaits ont des mères bachelières (toutes filières) mais aussi certifiées d'études primaires (graphique 5b). Également, les mères de niveau au moins bac +2 sont jugées les plus envahissantes dans le suivi scolaire (" elles s'en occupent trop »), mais elles sont suivies de près par les mères possédant au plus un CAP/BEP.

L'insatisfaction des adolescents à l'égard des pères les plus faiblement dotés en capital scolaire se perçoit plus précisément pour l'aide aux devoirs (graphiques $6 a$ et $6 b)$ : près de $40 \%$ des enfants de non diplômés et $30 \%$ des enfants dont les pères sont les plus faiblement dotés en capital scolaire (au plus le CAP/BEP) disent qu'ils ne les aident jamais dans les devoirs (contre respectivement $24 \%$ et $18 \%$ lorsqu'il s'agit des mères). A contrario les adolescents qui déclarent le plus fréquemment être « souvent " suivis par leurs pères sont des enfants de bacheliers (filière générale), puis de diplômés de $3^{e}$ cycle alors que pour les mères, cette modalité est choisie en priorité par des enfants de diplômées de $2^{\mathrm{e}}$ ou $3^{\mathrm{e}}$ cycle, puis par des bachelières (filière générale).
L'inégale compétence en savoirs et en méthodes scolaires selon le niveau d'études des parents joue donc un rôle indéniable, davantage pour le père que pour la mère, dans la manière dont les adolescents jugent l'aide parentale aux devoirs. Quand les parents sont non diplômés ou faiblement dotés en capital scolaire, l'insatisfaction concernant le suivi est plus forte à l'égard des pères qu'envers les mères, comme si ces dernières étaient présentes, s'investissaient malgré leurs manques de compétences scolaires, mobilisaient des dispositions favorables à la réussite scolaire. Ainsi Lahire (1995) a montré combien la réussite à l'école ne peut se réduire à un facteur explicatif, comme par exemple le niveau d'études des parents, preuve en est la réussite d'une part non négligeable d'enfants dont les parents ont de faibles niveaux d'études. D'autres dimensions contribuent à générer une configuration familiale consonante avec l'univers scolaire comme les formes familiales de culture écrite, les conditions d'exercice et les formes d'autorité parentale, les régularités temporelles et l'organisation domestique dans la famille, l'intérêt des parents pour la réussite sociale à travers la réussite scolaire, l'expérience parentale positive de l'école.

Par contre, les réponses des adolescents concernant la surveillance des résultats scolaires sont complètement indépendantes de leur milieu social d'origine (10) : les parents les moins dotés scolairement ne se distinguent pas par un intérêt moins grand à l'égard des performances de leur enfant à l'école. Ce résultat va à l'encontre de la conviction selon laquelle les familles les plus éloignées de l'univers scolaire, moins présentes à l'école, seraient les plus " démissionnaires " : la scolarité des enfants est un souci primordial pour la grande majorité des parents actuellement, quels que soient leur niveau scolaire ou leur situation sociale (Dubet, 1997).

\section{DES VARIATIONS SELON LA CATÉGORIE SOCIOPROFESSIONNELLE DES PARENTS}

Dans le domaine scolaire, les âges auxquels les élèves conçoivent devoir être autonome connaissent des variations selon la catégorie socioprofessionnelle (PCS) des parents qui sont trop peu significatives pour qu'elles soient sociologiquement interprétables. Par contre, l'estimation de l'aide apportée par les parents connaît des variations importantes, surtout du côté de la PCS du père. 
Graphique 7. - Estimation des adolescents concernant le suivi scolaire de leur père selon sa profession: "Penses-tu que ton père s'occupe suffisamment de ton travail scolaire ?"

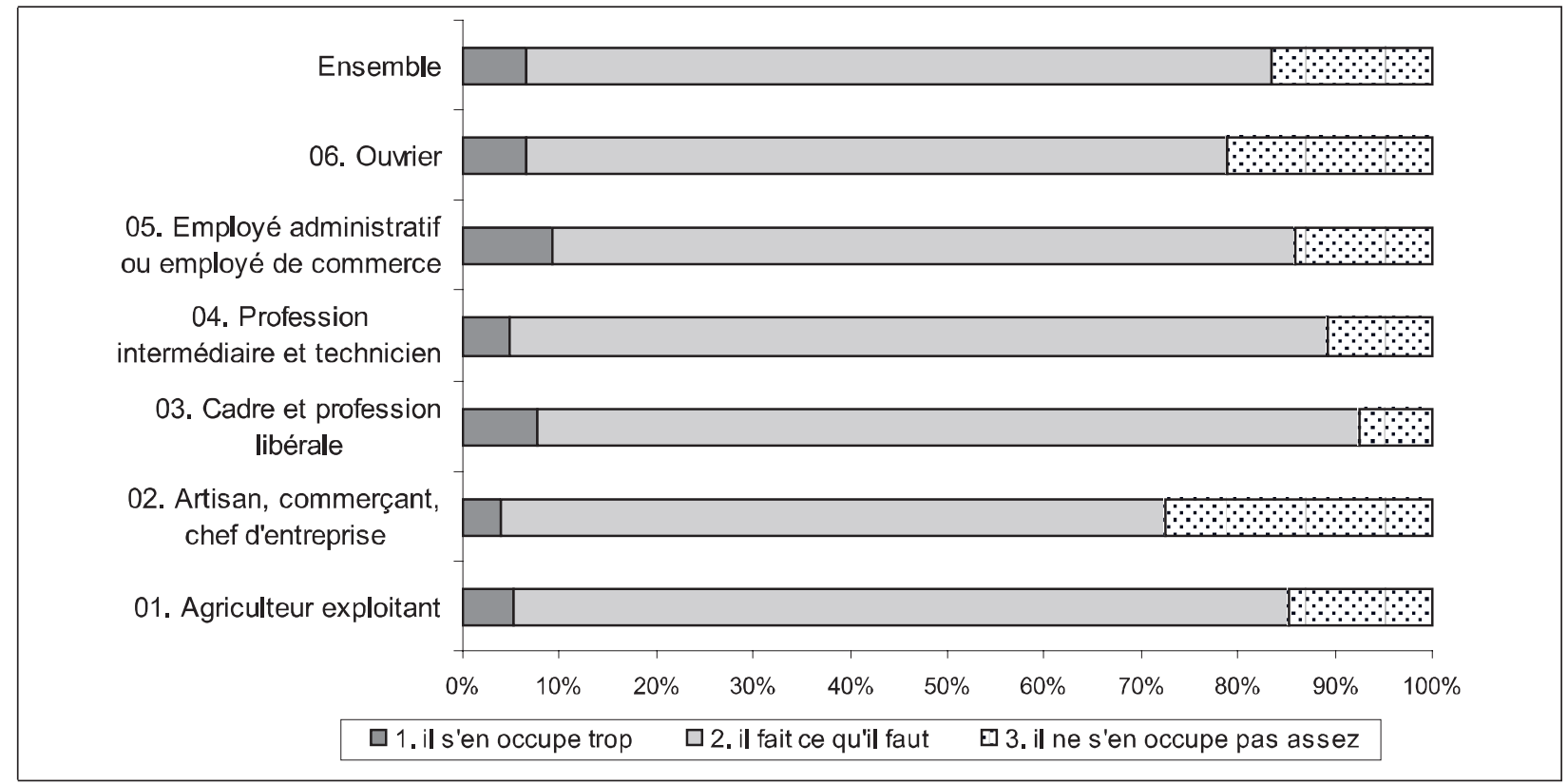

Légende : khi $2=20,8$; ddl = 10 (significatif à $5 \%$ ). Source : enquête PCV, partie "Éducation et famille 》 (INSEE, 2003). Note 1 : ont été éliminées les modalités "retraité ", " chômeur ", " autre personne sans activité professionnelle ", "sans objet » (père absent du foyer) dont chacune représente un effectif trop faible. Note 2 : ont été éliminées les modalités " ne sait pas ", "réponses inexploitables ", "non réponse ".

Les adolescents les plus satisfaits du suivi scolaire ont un père cadre/profession intellectuelle supérieure ou qui occupe une profession intermédiaire (graphique 7). Aucune variation significative n'est relevée par rapport à la situation socioprofessionnelle des mères pour cette question : est-ce parce que les mères s'occupent de toute façon de ce suivi, quelle que soit leur position professionnelle ? Les enfants dont le père est ouvrier sont plus insatisfaits du suivi scolaire que les autres, mais moins cependant que ceux dont le père est artisan, commerçant ou chef d'entreprise. Les parents de cette dernière catégorie professionnelle souffrent peut-être d'un manque de temps à consacrer à la vie privée et donc à l'éducation des enfants.

Les mères cadres/professions intellectuelles supérieures donnent l'impression d'être les moins interventionnistes : leurs enfants sont ceux qui estiment le plus être aidés " parfois " dans les devoirs (graphique $8 \mathrm{a}$ ) et bénéficier d'une surveillance maternelle des résultats scolaires à leur demande (graphique $8 \mathrm{~b}$ ). Deux interprétations non exclusives peuvent être avancées : les enfants ont tellement intériorisé les postures du travail scolaire inculquées très tôt qu'il n'est plus besoin pour les parents de les rappeler ou que ces enfants ne les ressentent plus comme des injonctions parentales. De ce point de vue, ils ont effectivement développé des dispositions propres à l'autonomie telle qu'elle est exigée à l'école aujourd'hui. Mais il peut s'agir également d'une conception relative à l'investissement masculin dans l'accompagnement scolaire : la mère peut se permettre d'être moins présente si le père intervient aussi dans le suivi scolaire. Dans l'enquête INSEE, lorsque les mères occupent une position de cadre et profession intellectuelle supérieure, les pères sont aussi dans cette situation dans plus de deux tiers des cas. Rappelons que les enfants dont le père est cadre/ profession intellectuelle supérieure sont les plus satisfaits de l'investissement paternel alors que les enfants de pères ouvriers ou artisans/commerçants/ chefs d'entreprise sont les plus insatisfaits (graphique 7). L'investissement dans l'accompagnement scolaire semble moins aller de soi du côté des ouvriers que des ouvrières (11) : les enfants de ces mères les trouvent aussi présentes que les autres pour l'aide aux devoirs (graphique 8a, question C1) 
Graphique 8a. - Estimation des adolescents concernant l'aide aux devoirs par leur mère : " $\mathrm{Si}$ tu as du travail scolaire à faire à la maison, es-tu aidé par ta mère ? " (question C1)

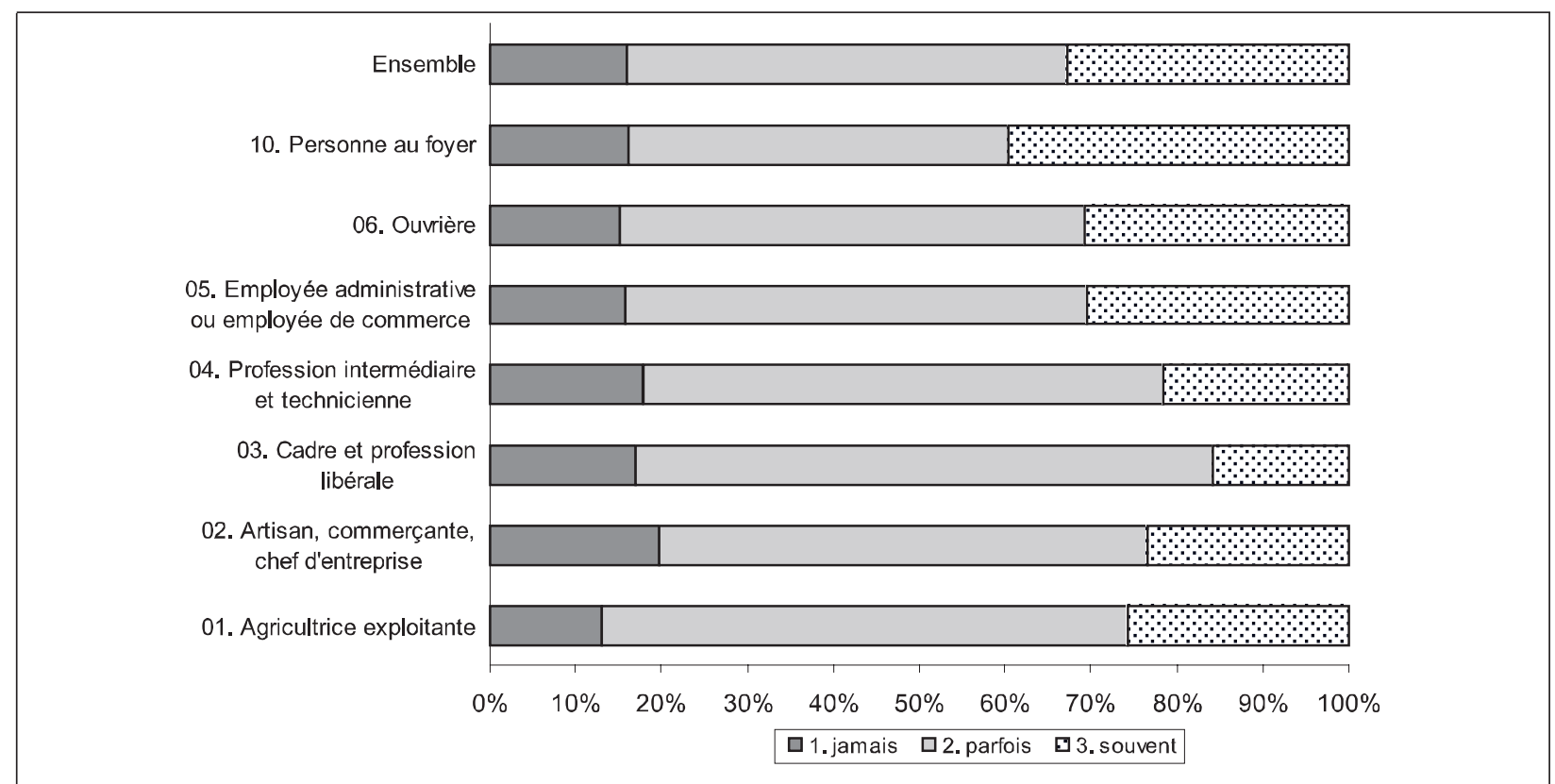

Légende : khi 2 = 23,35; ddl = 12 (significatif à 5 \%). Source : enquête PCV, partie " Éducation et famille » (INSEE, 2003).

Graphique 8b. - Estimation des adolescents concernant la surveillance des résultats scolaires par leur mère : "Comment ta mère surveille-t-elle tes résultats scolaires ? " (question C2)

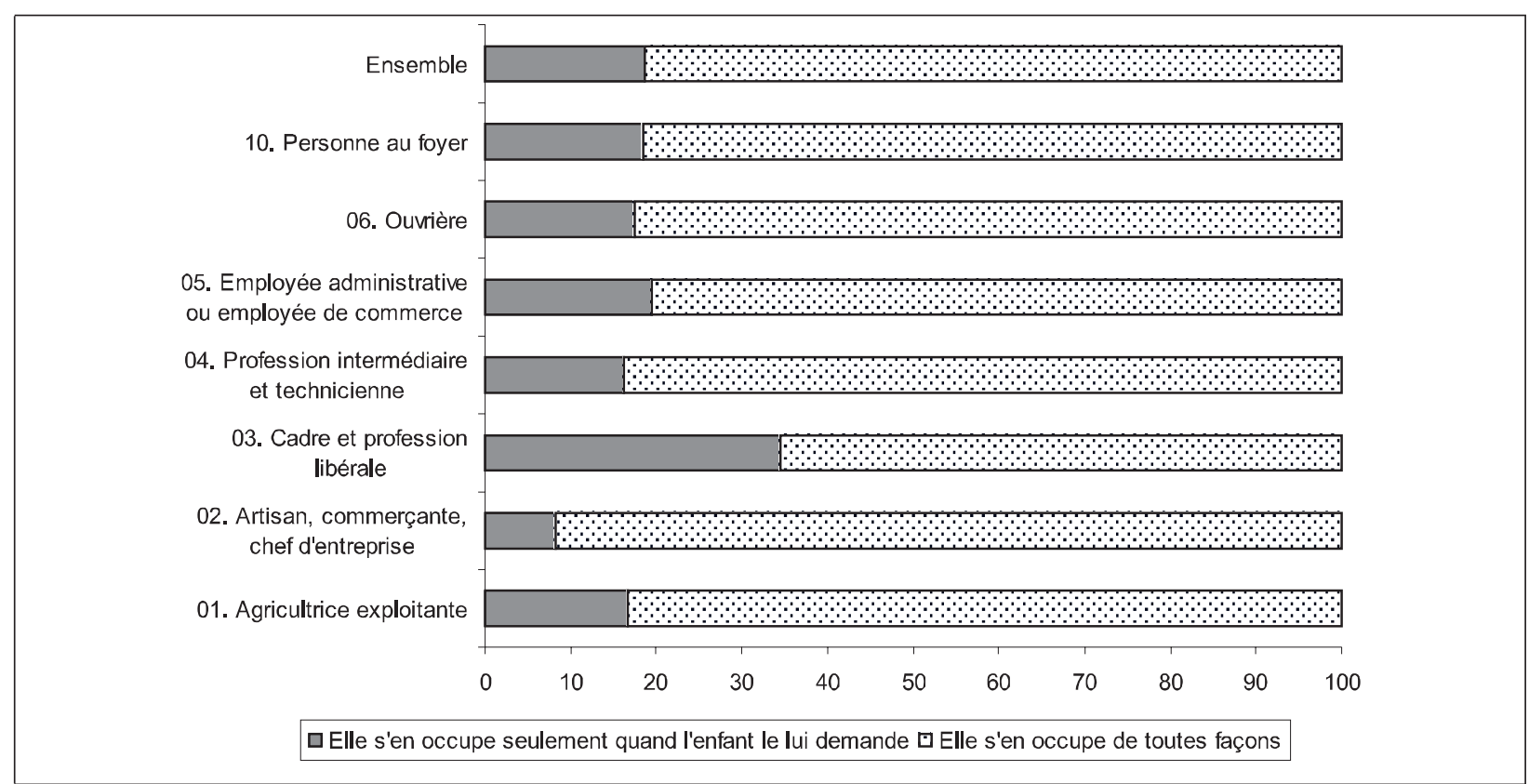

Légende : khi 2 = 14,7; ddl = 6 (significatif à $5 \%$ ). Source : enquête PCV, partie « Éducation et famille » (INSEE, 2003). 
et même un peu plus interventionnistes concernant les résultats scolaires (surveillance systématique plus grande et moins à la demande des enfants), en tout cas plus que les femmes cadres/professions libérales.

Enfin les propos des enfants sur leurs mères ne divergent pas fondamentalement selon qu'elles sont au foyer ou en activité professionnelle : à la question sur l'aide maternelle aux devoirs, on note un taux légèrement supérieur d'adolescents de mères au foyer estimant qu'ils en bénéficient « souvent » mais une proportion proche de la moyenne (un sur six) déclare qu'elles ne les aident "jamais ». Rien ne vient corroborer de manière nette l'impression selon laquelle les mères au foyer consacreraient plus de temps à la scolarité de leurs enfants que les mères qui travaillent. De ce point de vue, nos résultats confirment les conclusions de Letrait (2005) à propos des femmes au foyer qui consacrent globalement un temps plus long à leurs enfants de moins de 15 ans, sans que le temps alloué à l'éducation et aux jeux soit plus long que pour les mères en activité professionnelle : seuls augmentent les trajets et les soins aux enfants (les femmes au foyer ayant plus souvent que les autres un enfant de moins de 3 ans). En fait, le temps consacré aux jeux et à l'éducation (devoirs, etc.) par les mères dépend surtout de leur niveau d'étude, quel que soit leur statut d'occupation.

\section{CONCLUSION}

Les variations repérées dans les représentations des jeunes concernant l'autonomie dans le domaine scolaire sont finalement moins visibles au regard de la PCS des parents que si l'on considère les capitaux scolaires détenus par les parents. L'exemple du travail scolaire montre combien l'aspiration à l'autonomie est d'autant plus précoce que les adolescents peuvent bénéficier dans leur famille d'une aide (liée à des compétences scolaires estimées par des niveaux d'études dont on suppose qu'ils se traduisent en termes de contenus, d'habitudes) : plus la mère est dotée en capital scolaire, plus l'aspiration à l'autonomie du collégien/lycéen est forte. Par contre, la perception de l'investissement dans la scolarité par les mères est moins dépendante des capitaux maternels objectivement détenus, ce qui peut s'interpréter par un suivi important de la scolarité de l'enfant quel que soit le niveau d'études des mères (alors que les enfants semblent souffrir d'un investissement moindre quand le père est peu doté en capital scolaire), mais aussi comme l'effet d'une intériorisation suffisamment forte dans certains milieux sociaux de l'injonction parentale à être scolairement autonome.

Ces résultats sont importants à considérer à une époque où l'école valorise beaucoup l'autonomie de l'élève : il s'agit bien d'une compétence dont la construction est contextualisée et socialement différenciée. II n'existe pas une seule forme d'autonomie, comprenant les mêmes exigences, se déclinant selon les mêmes modalités, mais bien plusieurs autonomies suivant les domaines concernés (habillement, fréquentations amicales, usage des loisirs, utilisation de son argent de poche, scolarité...), avec des conséquences socialement différenciées (la renommée auprès des pairs, la réussite scolaire). L'autonomie d'un adolescent à l'école n'est pas une aspiration «naturelle ", elle requiert des conditions d'indépendance réelles et précises (savoir s'orienter dans l'univers scolaire, décoder correctement les attentes cognitives et comportementales, avoir des habitudes de travail, ne pas être trop démuni face aux demandes didactiques...). La socialisation des filles apparaît plus congruente avec les dispositions scolaires attendues, ce qui expliquerait leurs représentations plus précoces de l'accès à l'autonomie concernant le travail scolaire. Enfin, l'autonomie dans le cadre de l'école s'acquiert d'autant plus facilement que les parents ont à la fois une certaine familiarité avec l'univers scolaire et une mobilisation active de leurs capitaux pour épauler leur enfant. Souvent ces deux conditions familiales ne sont pas réunies pour assurer un étayage scolaire suffisant. II appartient alors à l'école d'être vigilante quant à l'accompagnement pratique, concret et visible des comportements de l'élève qui lui permettent de développer une autonomie scolairement reconnue et efficace du point de vue des apprentissages. Nous rejoignons par là les conclusions de $\mathrm{S}$. Bonnéry (2007).

Rachel Gasparini rachel.gasparini@iufm.univ-lyon1.fr GRS, Université de Lyon et IUFM de l'académie de Lyon, université Claude-Bernard-Lyon 1

Odile Joly-Rissoan odile.joly@univ-savoie.fr LLS, université de Savoie

Monique Dalud-Vincent monique.vincent-dalud@univ-lyon2.fr GRS, Université de Lyon 
(1) Au collège, depuis la rentrée 2006, l'autonomie de l'élève en tant que compétence visée par le socle commun de connaissances fait l'objet d'une évaluation dans le cadre de la " note de vie scolaire ", qui compte dans le contrôle continu pour le brevet des collèges et dont la mise en place n'est pas sans susciter quelques remous dans les établissements.

(2) Notre équipe a eu accès aux données à une époque où elles n'étaient pas encore téléchargeables librement. Suite à un appel d'offres de I'INSEE qui demandait aux équipes de proposer un plan de traitement des données, notre projet a été retenu avec pour objectif d'analyser les variations dans les représentations de pour objectif d'analyser les variations dans les représentati 作 lycéens.

(3) La question F14: «À quel âge un enfant ou un jeune devrait avoir le droit d'organiser soi-même son travail scolaire ? " a été particulièrement analysée, sachant qu'elle n'est pas exempte d'interprétations multiples de la part des enquêtés dans le sens d'un droit mais aussi d'un devoir. Ces deux dimensions sont d'ailleurs très intriquées : un enfant qui a tellement intériorisé un "devoir " peut le transformer en nécessité intérieure qui n'est plus un devoir extérieur.

(4) Le test de Mann-Withney, adapté pour comparer des réponses à des variables quantitatives appariées, a permis de montrer que seules deux questions obtenaient des réponses significativement plus faibles que F14 : F12 ("choisir ses émissions de télé ») et F9 (" choisir tout seul ses vêtements dans les magasins"). Nous avons dû utiliser ce test dans la mesure où aucune variable de F5 à F15 ne suivait une loi normale. Le test recherche si, statistiquement parlant, on peut affirmer que, dans la population, plus d'un adolescent sur deux a répondu un âge plus grand pour l'une des données appariées (comparaison des réponses d'un même lescent), parfois sur des données non appariées. On parlera de " domination " de $x$ sur y pour dire que le test est significatif à
$5 \%$ et donc que les adolescents ayant la caractéristique $x$ répondent plus souvent un âge plus élevé que ceux ayant la caractéristique y. Les analyses qui suivent en termes de " domination » utiliseront ce test, sans que sa référence soit systématiquement rappelée.

(5) Nous entendons par genre " la dimension sociale des rôles associés aux individus de sexe féminin et masculin " (Dafflon Novelle, 2006, p. 10).

(6) Cousin et Felouzis (2002) soulignent combien la nouveauté dans l'arrivée au collège ne relève pas tellement des contenus mais de la socialisation. L'école primaire garde une nature de " communauté ", les relations entre élèves et maîtres sont plus fortement chargées d'éléments affectifs forts qu'au collège où un processus de distanciation se met en place.

(7) Les garçons collégiens sont plus contrôlés par leurs parents que les filles dans leurs devoirs et activités scolaires (Bounoure, 1995).

(8) Les niveaux de diplôme des parents ont été regroupés en 7 groupes. Groupe 1 : aucun diplôme ; groupe 2 : certificat d'études primaires; groupe 3 : CAP/BEP ; groupe 4 : bac technique, professionnel, brevet de technicien; groupe 5 : bac général ; groupe 6 : diplôme universitaire de $1^{\text {er }}$ ou de $2^{\mathrm{e}}$ cycle: groupe 7 : diplôme universitaire de $3^{\mathrm{e}}$ cycle.

(9) À une exception près : les enfants dont le père possède un diplôme de $2^{\mathrm{e}}$ cycle.

(10) On n'observe pas de différences significatives dans les réponses des enfants selon la PCS de leurs parents à la question : "Comment tes parents surveillent-ils tes résultats scolaires?"

(11) Toujours dans cette enquête INSEE, lorsque les mères sont ouvrières, les pères sont ouvriers dans plus de deux tiers des cas.

\section{BIBLIOGRAPHIE}

BAUDELOT C. \& ESTABLET R. (2007). Quoi de neuf chez les filles ? Entre stéréotypes et libertés. Paris : Nathan.

BAUTIER É. \& ROCHEX J.-Y. (1998). L'expérience scolaire des nouveaux lycéens. Démocratisation ou massification ? Paris : Armand Colin.

BONNÉRY S. (2007). Comprendre l'échec scolaire. Élèves en difficultés et dispositifs pédagogiques. Paris : La Dispute.

BOUNOURE A. (1995). Parents de collégiens. Conceptions et pratiques éducatives. Paris : INRP.

CONSEIL DE L'EMPLOI, DES REVENUS ET DE LA COHÉSION SOCIALE (2002). Éducation et redistribution, rapport $n^{\circ}$ 3. Paris : La Documentation française. Disponible sur Internet à l'adresse : <http://www.prospective-foresight.com/IMG/pdf/rapport3cerc.pdf> (consulté le 10 décembre 2009).

COUSIN O. \& FELOUZIS G. (2002). Devenir collégien. L'entrée en classe de sixième. Issy-les-Moulineaux: ESF.

DAFFLON NOVELLE A. (2006). Filles-garçons. Socialisation différenciée ? Grenoble : Presses universitaires de Grenoble.

DAMON J. (2004). « Les adolescents : données de cadrage ". Recherches et prévisions, $\mathrm{n}^{\circ} 76$.

DUBET F. (1997). École, familles : le malentendu. Paris : Éd. Textuel.
DURU-BELLAT M. (1994). "Filles et garçons à l'école, approches sociologiques et psycho-sociales. $1^{\text {re }}$ partie : des scolarités sexuées, reflet de différences d'aptitude, ou de différences d'attitudes ? ". Revue française de pédagogie, $\mathrm{n}^{\circ} 109$.

DURU-BELLAT M. (1995). "Filles et garçons à l'école, approches sociologiques et psycho-sociales. $2^{\mathrm{e}}$ partie : la construction scolaire des différences entre les sexes ". Revue française de pédagogie, $\mathrm{n}^{\circ} 110$.

ELIAS N. (1985). La société de cour. Paris : Flammarion.

ELIAS N. (1991). La société des individus. Paris : Fayard.

ELIAS N. (1993). Engagement et distanciation. Paris : Fayard.

GASPARINI R. (1998). La discipline à l'école primaire. Une interprétation sociologique des modalités d'imposition de l'ordre scolaire. Thèse de doctorat, sociologie et sciences sociales, université Lumière-Lyon 2.

GOUYON M. (2004). «Années scolaires 1991-1992 et 20022003. L'aide aux devoirs apportée par les parents". INSEE Première, $\mathrm{n}^{\circ} 996$.

HAMELINE D. (1999). «Autonomie ». Questions pédagogiques. Paris : Hachette.

HENRI-PANABIÈRE G. (2007). Collégiens en difficultés scolaires issus de parents fortement diplômés. Analyse des composantes du capital culturel et des conditions de sa 
transmission. Thèse de doctorat, sociologie et sciences sociales, université Lumière-Lyon 2.

INSEE (2003). «Enquête "Éducation et famille" 2003 ». Disponible sur Internet à l'adresse : <http://www.insee.fr/ $\mathrm{fr} /$ themes/detail.asp?reg id=0\&ref $i d=f d$-edufa03> (consulté le 10 décembre 2009).

KEDDIE N. (2007). «Le savoir dispensé dans la salle de classe ». In J. Deauvieau \& J.-P. Terrail, Les sociologues, l'école \& la transmission des savoirs. Paris : La Dispute.

KELLERHALS J. \& MONTANDON C. (1991). Les stratégies éducatives des familles. Milieu familial, dynamique familiale et éducation des pré-adolescents. Paris : Delachaux \& Niestlé.

LAHIRE B. (1995). Tableaux de familles. Paris : Gallimard et Éd. du Seuil.

LAHIRE B. (2001). " La construction de l'“autonomie” à l'école primaire : entre savoirs et pouvoirs ". Revue française de pédagogie, $\mathrm{n}^{\circ} 134, \mathrm{p} .151-161$.
LEPOUTRE D. (1997). Cœur de banlieue. Codes, rites et langages. Paris: Odile Jacob.

LETRAIT M. (2005). « Temps consacré aux enfants, au travail et moments pour soi des mères ". Recherches et prévisions, $\mathrm{n}^{\circ} 82$.

MILLET M. \& THIN D. (2005). Ruptures scolaires. L'école à l'épreuve de la question sociale. Paris : PUF.

MEN (2005). « Loi d'orientation et de programme pour l'avenir de l'école ». Loi n² 2005-380 du 23 avril 2005. Bulletin officiel de l'Éducation nationale, 5 mai 2005, $\mathrm{n}^{\circ} 18$.

MEN (2006). « Socle commun de connaissances et de compétences ». Décret $n^{\circ} 2006-830$ du 11 juillet 2006. Bulletin officiel de l'Éducation nationale, 20 juillet 2006 , $\mathrm{n}^{\circ} 29$.

PLAISANCE É. (1986). L'enfant, la maternelle, la société. Paris : PUF.

SINGLY F. de (2006). Les adonaissants. Paris : Armand Colin.

VINCENT G. (1980). L'école primaire française. Lyon : Presses universitaires de Lyon. 


\section{Annexe. - Note méthodologique}

L'enquête "Éducation et famille » constitue la partie variable de l'« Enquête permanente sur les conditions de vie des ménages " (EPCV) réalisée par I'INSEE en octobre 2003, auprès de 4100 ménages ayant au moins un enfant âgé de 2 à 25 ans et scolarisé. Les questions concernaient la vie de famille, les parents et les enfants. Un questionnaire était destiné aux parents mais également à l'enfant du ménage, tiré au sort, lorsqu'il était collégien ou lycéen. L'échantillon porte sur 1405 adolescents âgés de 10 à 23 ans et scolarisés dans un établissement secondaire en octobre 2003.

Toute recherche portant sur les catégories intermédiaires telles que les « adolescents » ou les « jeunes " est confrontée à la question de la définition des tranches d'âge qui permettent de déterminer la population à interroger. Par exemple si l'ONU retient la tranche d'âge 10-19 ans pour l'adolescence, d'autres (comme la Conférence de la famille en 2004 en France) considèrent qu'elle commence à l'âge " normal » d'entrée au collège (11 ans) et qu'elle se termine à l'âge de la majorité légale française (17 ans révolus), d'après Damon (2004). Ici I'INSEE a fait le choix de parler d'adolescents sélectionnés à partir de leur position institutionnelle scolaire : la scolarité est une expérience fondamentale pour un individu dans nos sociétés occidentales actuelles, mais cette option écarte les adolescents qui ne sont plus scolarisés (au-delà de 16 ans), qui sont « déscolarisés » ou qui ont arrêté leurs études. Or ces arrêts d'études ne sont pas distribués de manière égale dans la population française puisqu'en 2002, si près d'un jeune de plus de 18 ans sur cinq a arrêté ses études, presque $30 \%$ d'enfants d'ouvriers sont concernés contre $5 \%$ d'enfants de cadres (d'après le rapport "Éducation et redistribution ", Conseil de l'emploi, des revenus et de la cohésion sociale, 2002). Nos interprétations ne sont donc pas généralisables à l'ensemble des adolescents, mais se restreignent à ceux qui sont scolarisés en collège ou en lycée (général, technologique, professionnel). À l'inverse, nos interprétations s'étendent à des individus qui, compte tenu de leurs âges (entre la majorité légale en France et 23 ans), seraient considérés dans d'autres recherches plutôt comme de «jeunes " étudiants mais qui, du fait de leur scolarisation dans le secondaire, se retrouvent classés ici dans les « adolescents ».

La variable F14 fait partie d'un ensemble correspondant à la question : "À ton avis, à quel âge environ un enfant ou un jeune devrait avoir le droit de faire les choses suivantes ? ". Les réponses à cette question sont considérées comme des indicateurs des représentations que se font les adolescents de l'âge auquel on peut être autonome. Les domaines concernés sont :

- F5 : « Rester seul un soir à la maison en l'absence de ses parents ";

- F6 : «Inviter des amis ou des amies à la maison sans demander l'avis des parents ";

- F7 : «Passer la nuit chez des amis ou des amies »;

- F8 : «Aller au cinéma sans les parents »;

- F9 : «Choisir tout seul ses vêtements dans les magasins ";

- F10 : «Décider de la façon de dépenser son argent »;

- F11 : «Avoir un compte en banque ou un compte à la Poste »;

- F12 : «Choisir ses émissions de télé »;

- F13 : «Choisir ses jeux vidéo ou les sites qu’il visite sur internet »;

- F14 : «Organiser soi-même son travail scolaire » ;

- F15 : «Décider de l'heure de son coucher».

Enfin d'autres questions ont été analysées:

- C1 : «Si tu as du travail scolaire à faire à la maison, es-tu aidé par : ta mère, ta belle-mère, ton père, ton beau-père, un frère ou une sœur? ";

- C2 : «Comment tes parents surveillent-ils tes résultats scolaires? »;

- C3 : « Penses-tu que tes parents s'occupent suffisamment de ton travail scolaire ? »:

- Ta mère/belle-mère, ton père/beau-père ne s'en occupe pas ;

- Elle/il s'en occupe seulement quand tu lui demandes ;

- Elle/il s'en occupe de toute façon. 